\title{
Strengthening Community Surveillance of Ebola Virus Disease in Sierra Leone
}

\author{
AnhMinh A. Tran*, Adam Hoar, Alyssa J. Young, Allison Connolly, Mary-Anne Hartley, \\ Samuel Boland, Brooke Mancuso, Guddu Kaur, John Esplana, Erin Polich and Laura \\ Fisher
}

Epidemiology and Surveillance, GOAL Global, Fairfax, VA, USA

\section{Objective}

Systematically assess and strengthen the capacity of communities and sections in Port Loko District, Sierra Leone to detect significant events related to the reporting of Ebola virus disease (EVD) such as sick persons, secret burials and deaths. The components of the enhanced surveillance system will be described.

\section{Introduction}

Communities and sections that are consistently underreporting both illness and death pose a significant risk to surveillance and their efficacy is dependent upon the reporting of community structures such as government structures (primary health units (PHUs), schools), EVD response structures (contact tracers, community events based surveillance (CEBS), social mobilization), and traditional structures (chiefs, traditional healers, village task forces, religious institutions).

All structures are required to report to the District Ebola Response Center (DERC) as depicted in Figure 1.

Frequent and protocolized information sharing is central to the reporting efficacy within this structure to ensure early capture of all EVD-related incidents.

\section{Methods}

Underreporting in 162 sections and communities in Port Loko district was determined by using a proxy of standard mortality ratio $(\mathrm{SMR}=$ reported deaths/expected deaths). Port Loko estimated mortality data (17.1 deaths per 1000/year) and population figures from the Statistics Sierra Leone were used to calculate expected deaths. Reported deaths were gathered through the national 117 alert system and verified by surveillance teams and burial teams. Sections were categorized by reporting efficacy (ranging from $0 \%$ to $100+\%$ of expected deaths reported). Sections with the lowest reporting efficacies were prioritized first. Systematic interviews with each community reporting structures (government, EVD response and traditional). Interviews were conducted using an Open Data Kit (ODK) smartphone based closed questionnaire, designed to cross-check EVD reporting statistics and actions recorded under each reporting structure. Responses will be compared for levels of consensus (weighted scales and kappa scores) to evaluate the efficacy of the communication network and frequency of information sharing between reporting structures. Data are being used to find the barriers to reporting (e.g. leadership structure, awareness campaigns and geographic boundaries) and identify areas of weakness to provide a tailored response to strengthening surveillance.

\section{Results}

The described active surveillance model was implemented on June 15,2015 . Sick and death alerts were compared 4 weeks before the implementation of the active surveillance model and 4 weeks after implementation. In the 4 week period before implementation, Port Loko had 136 sick alerts and 749 death alerts, compared with 334 sick alerts and 869 death alerts in the 4 weeks following, a $146 \%$ and $16 \%$ increase respectively, for an overall increase of $36 \%$. This increase in reporting was particularly pronounced in sections that previously underreported.

\section{Conclusions}

Focusing active surveillance efforts on silent sections leads to increased reporting of significant EVD events and the development of an integrated section-level surveillance system to identify disease triggers. Finally, strengthening disease-reporting structures may also support the detection of other rare and rapidly propagating infectious diseases, which may improve the reporting capacity of more common endemic disease and thus have wider benefits on the health system.

Further analysis of the active surveillance questionnaires to assess consensus among reporting structures is still ongoing and central to maintaining the integrity of EVD surveillance.

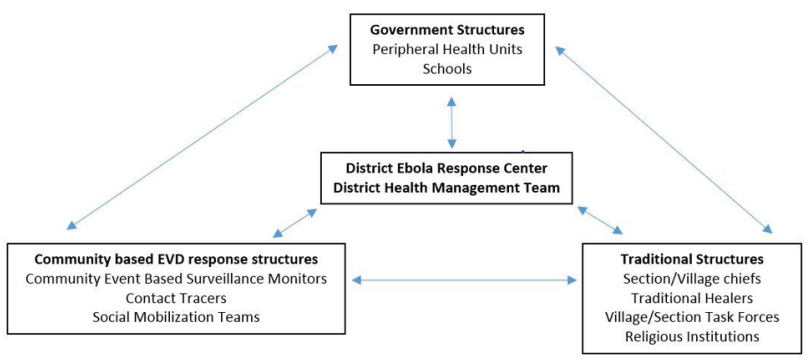

Figure 1. Relationship of Reporting Stuctures

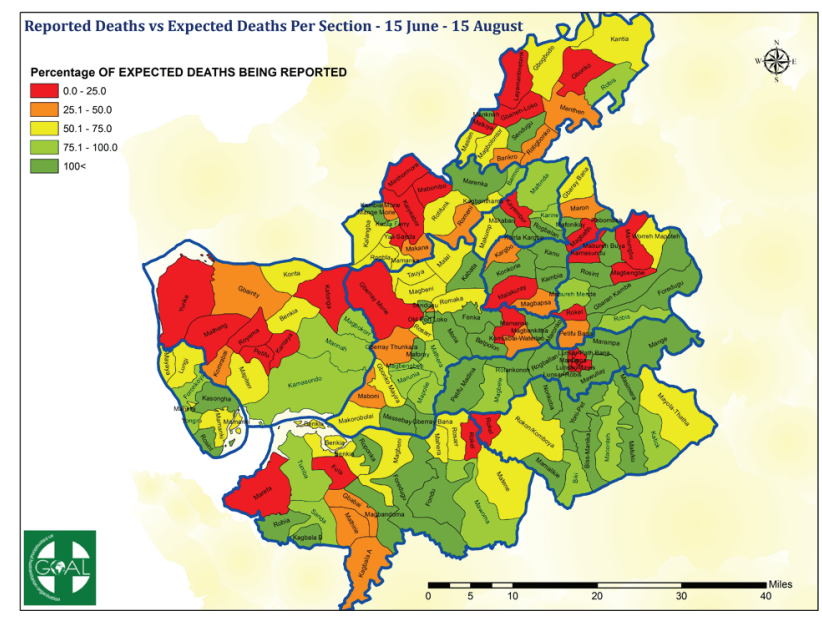

Figure 2. Reported deaths vs Expected deaths by Section

Keywords

surveillance; ebola; Epidemiology

\section{Acknowledgments}

GOAL would like to acknowledge the contributions of the Port Loko District Health Medical Team, District Ebola Response Center, WHO, CDC, Unicef

\section{${ }^{*}$ AnhMinh A. Tran}

E-mail: aat775@gmail.com 